\title{
Determining the relationship between the refractive-index difference of a coiled single-mode optical fiber and its bending radius by a mode-image analysis method
}

Fang-Wen Sheu, Yi-Syuan Lu

Fang-Wen Sheu, Yi-Syuan Lu, "Determining the relationship between the refractive-index difference of a coiled single-mode optical fiber and its bending radius by a mode-image analysis method," Proc. SPIE 9793, Education and Training in Optics and Photonics: ETOP 2015, 97931C (8 October 2015); doi: $10.1117 / 12.2223107$

SPIE Event: Education and Training in Optics and Photonics: ETOP 2015, 2015, Bordeaux, France 


\title{
Determining the relationship between the refractive-index difference of a coiled single-mode optical fiber and its bending radius by a mode-image analysis method
}

\author{
Fang-Wen Sheu*, Yi-Syuan Lu \\ Department of Electrophysics, National Chiayi University, Chiayi 60004, Taiwan
}

\begin{abstract}
In this experiment, we wrap a single-mode optical fiber around various sizes of cylindrical acrylic tubes, forming a series of manually controllable birefringent optical fibers. We launch linearly polarized external-cavity feedback wavelength-tunable visible diode laser light into the stressed birefringent fiber and capture simultaneously the fiber cross-sectional images of two orthogonally polarized modes under various wavelengths by a Wollaston prism polarizer. Through image analysis, we can obtain the two orthogonally polarized light intensities and calculate the phase difference of the two orthogonally polarized modes in the fiber core. By examining the variations of the phase difference with the optical wavelength, we can thus obtain the refractive-index difference of the birefringent fiber. Then by successively changing the size of the acrylic tube, we demonstrate that the refractive-index difference of a coiled fiber varies with its bending radius in a square inverse law. This experiment can reveal precisely the variations in the birefringence of a coiled fiber by a home-made wavelength-tunable diode laser and a mode-image analysis method, providing an advanced teaching kit in the optics laboratory.
\end{abstract}

Keywords: Single-mode optical fiber, birefringent optical fiber, acrylic tube, wavelength-tunable diode laser, fiber cross-sectional image, refractive-index difference, bending radius

\section{INTRODUCTION}

A single-mode optical fiber can become a birefringent (or polarization-maintaining) optical fiber if we change its geometrical configuration by bending, twisting, or compressing it [1,2]. The propagation constants of the two modes orthogonally polarized at the two principle axes of a bent fiber will differ from each other. Wavelength sweeping (or scanning) is a simple and fast method to determine the refractive-index difference $\Delta n$ of a birefringent fiber [3-7]. We have constructed an external-cavity feedback wavelength-tunable diode laser light source [8] and measured the $\Delta n$ of a birefringent fiber from the wavelength dependence of the phase difference between the two orthogonally polarized modes by analyzing the fiber cross-sectional images at the fiber output end [6]. In this report, we wrap a single-mode optical fiber around various sizes of cylindrical acrylic tubes to form a series of birefringent optical fibers with manually controllable $\Delta n$ values. Then we determine the relationship between the $\Delta n$ of a coiled single-mode optical fiber and its bending radius $R$ [9]. This experimental system can measure precisely the variations in the birefringence of a coiled fiber by a home-made wavelength-tunable diode laser and a fiber mode-image analysis method. It demonstrates an advanced teaching kit in the optics laboratory.

\section{EXPERIMENTAL SETUP AND METHOD}

Figures 1 and 2 show the schematic diagram and photograph of the experimental setup. Figure 3 shows the photograph of the home-made red diode laser (Union Optronics U-LD-650551A) light source and its driving system. We take a single-mode optical fiber (Thorlabs SM600, core diameter $4.45 \mu \mathrm{m}$, cladding diameter $125 \mu \mathrm{m}$, cut-off wavelength $550 \pm$ $50 \mathrm{~nm}$ ) to wrap around a cylindrical acrylic tube with some outer radius for several turns, formig a manually controllable birefringent optical fiber. The fiber birefringence is related the size of the cylindrical acrylic tube (Figure 4). The output beam of the linearly polarized diode laser is first launched to a cubic beam splitter. The transmitted beam from the beam splitter, having a polarization angle of $45^{\circ}$ with respect to the principal axes of the stressed birefringent fiber, is launched

*fwsheu@mail.ncyu.edu.tw; phone +886 5 271-7993; fax +886 5 271-7909

Education and Training in Optics and Photonics: ETOP 2015, edited by Eric Cormier, Laurent Sarger Proc. of SPIE Vol. 9793, 97931C · C 2015 SPIE, IEEE, OSA, ICO · doi: 10.1117/12.2223107 
into the test fiber input end via a focusing 20X microscope objective (MO) lens. The emerging light at the test fiber output end is projected onto a CCD camera (Newport LBP-3-USB) by an imaging 10X MO lens. A Wollaston prism polarizer (CVI WLST-5.0-CA-425-675) is then put in front of the CCD camera, thus we can observe simultaneously the fiber cross-sectional images of the two orthogonally polarized modes in the bent fiber core [6]. Through fiber mode-image analysis, we can obtain the two orthogonally polarized mode light intensities in the bent fiber core from a single snapshot image. This arrangement can inhibit effectively the power instability problem of an external-cavity feedback wavelength-tunable diode laser [8], since the calculation of the phase difference of the two orthogonally polarized modes depends on the ratio of the intensities of these two modes [3].

The reflected beam from the beam splitter is directed to a ruled reflective diffraction grating (Thorlabs GR25-1850, Blaze wavelength $500 \mathrm{~nm}, 1800$ Grooves $/ \mathrm{mm}$ ), which can diffract the light of a specific wavelength back to the beam splitter and also partly back to the laser diode. The external-cavity optical feedback can cause the diode laser to become lasing at this specific wavelength [8]. Therefore the optical wavelength of the external-cavity feedback diode laser can be tuned by slightly varying the diffraction grating's angle. The diffracted light passing through the beam splitter is coupled into a pigtail fiber by a 20X MO lens, which can guide the laser light to an optical spectrum analyzer (Advantest Q8384) to monitor the output spectrum of the diode laser.

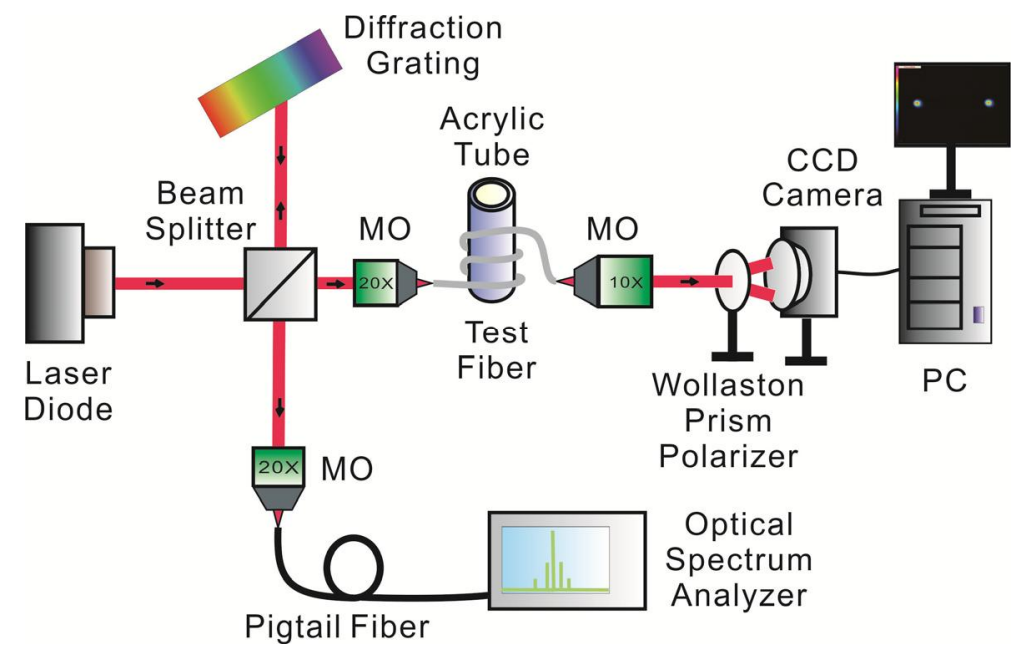

Figure 1. The schematic diagram of the experimental setup.

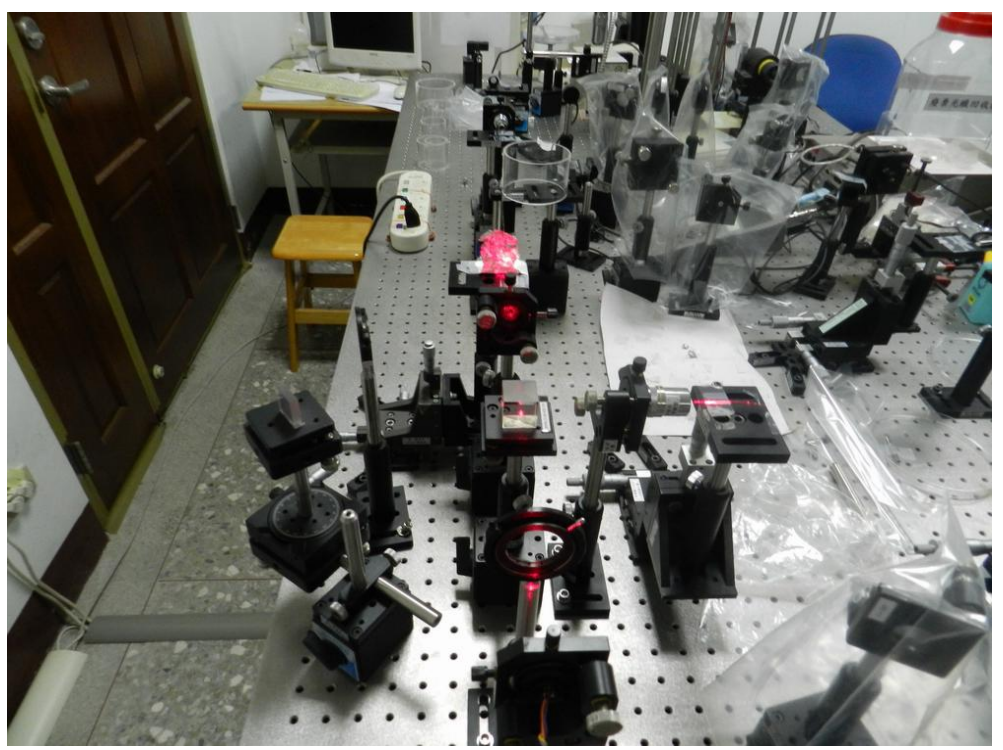

Figure 2. The photograph of the experimental setup. 


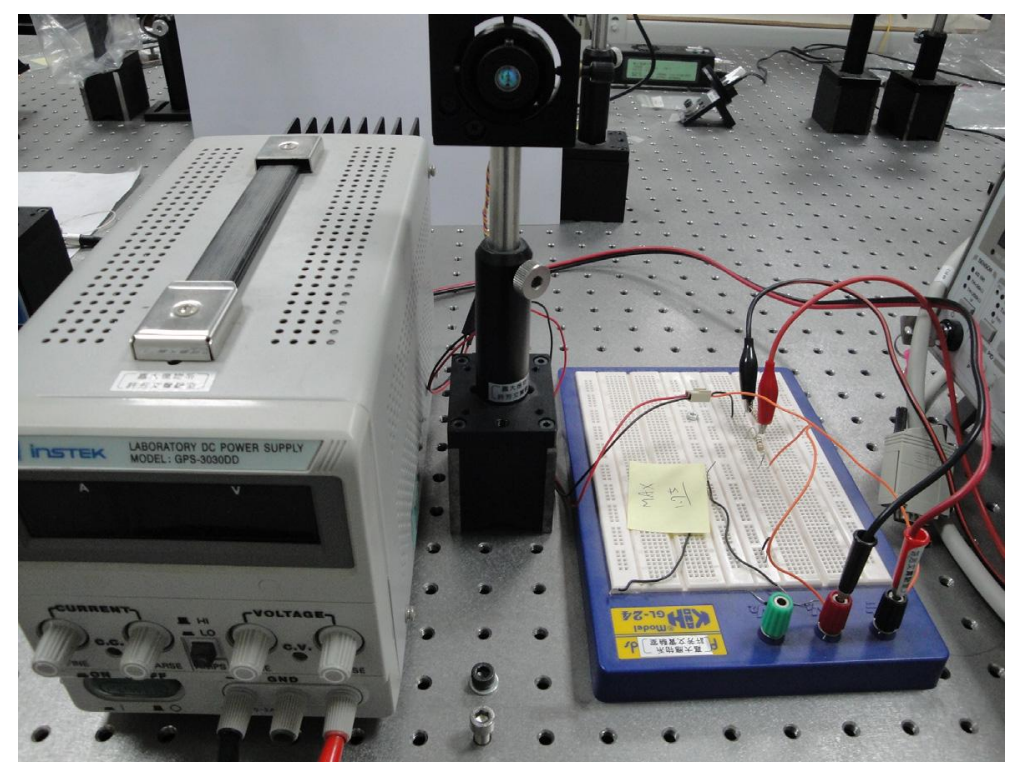

Figure 3. The photograph of the diode laser light source and its driving system.

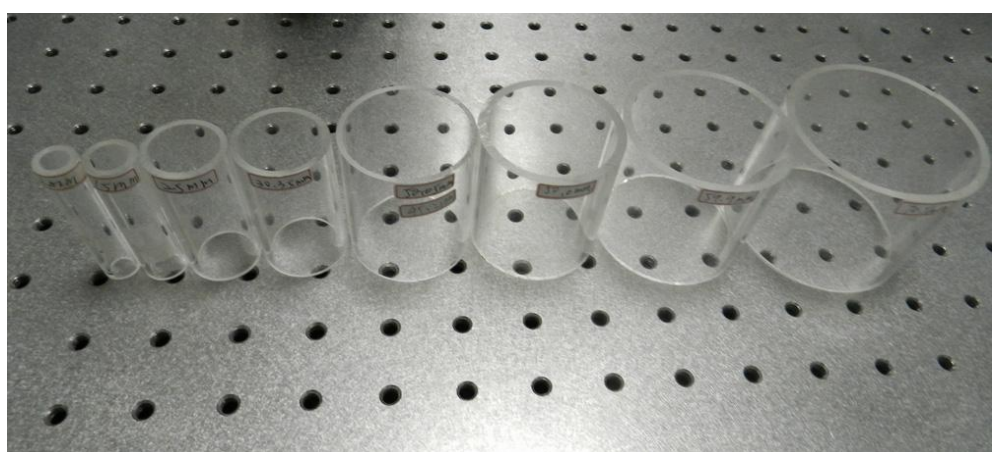

Figure 4. The photograph of the various sizes of cylindrical acrylic tubes.

\section{EXPERIMENTAL RESULTS}

For an example, we first take a single-mode optical fiber (Thorlabs SM600) to wrap around a cylindrical acrylic tube with an outer radius of $12.50 \mathrm{~mm}$ for three turns. We launch the linearly polarized external-cavity feedback wavelength-tunable red diode laser light beam into the stressed birefringent fiber. As demonstrated in Figure 5, by a Wollaston prism polarizer and a tunable diffraction grating, we can capture simultaneously the fiber cross-sectional images of two orthogonally polarized modes under various wavelengths.

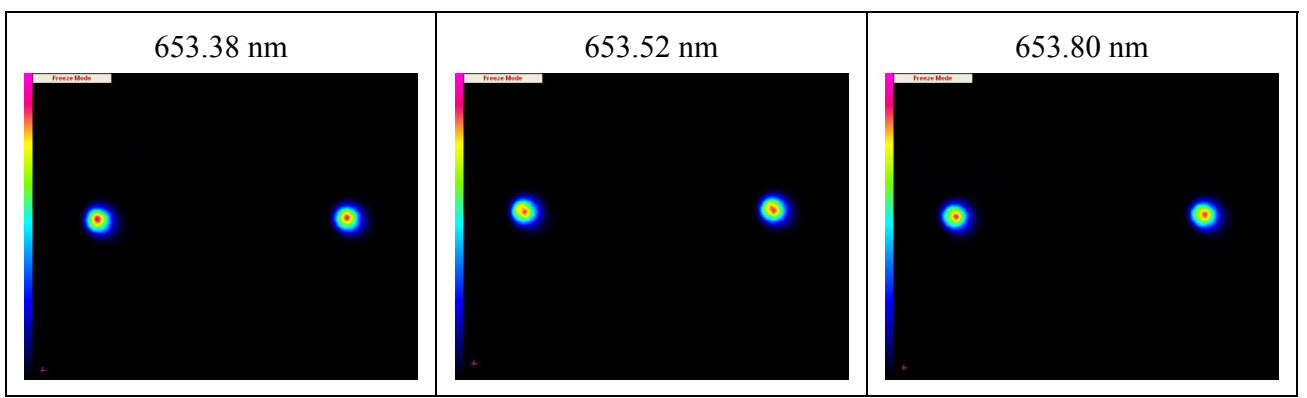




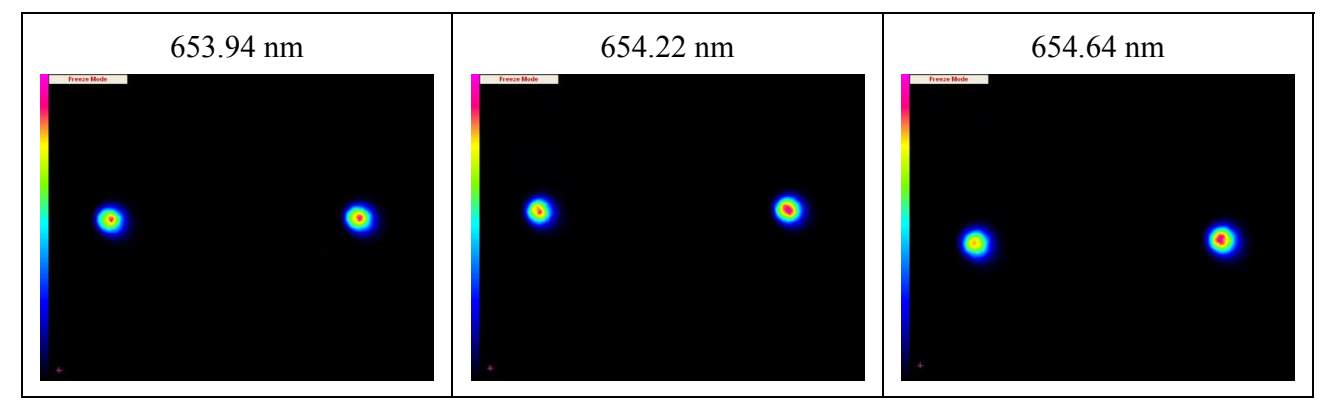

Figure 5. The fiber cross-sectional images of the two orthogonally polarized modes in a coiled single-mode optical fiber with a bending radius of $12.50 \mathrm{~mm}$ under various wavelengths.

By analyzing individually the orthogonally polarized fiber mode images and calculating the total light intensity around the fiber core (ignoring the background light in the fiber cladding), we can obtain separately the intensities $I_{a}$ and $\mathrm{I}_{\mathrm{b}}$ of the two orthogonally polarized modes in a coiled single-mode optical fiber from a single snapshot image. Thus, the phase difference $\phi$ between the two modes can be calculated by the formula $\sin \phi= \pm 2 \sqrt{\mathrm{I}_{\mathrm{a}} \mathrm{I}_{\mathrm{b}}} /\left(\mathrm{I}_{\mathrm{a}}+\mathrm{I}_{\mathrm{b}}\right)$ [3]. By tuning the diffraction grating's angle step by step, we can measure the variation of the phase difference $\phi$ as a function of the wavelength $\lambda$, and then calculate the $\phi-\lambda$ relationship. For an example, Figure 6 shows the measured phase difference of the two orthogonally polarized modes under various wavelengths for a coiled fiber with a bending radius of $12.50 \mathrm{~mm}$, which gives $\Delta \phi / \Delta \lambda=-2.63381$ degree $/ \mathrm{nm}$ from a linear fitting curve.

The phase difference of the two modes can be expressed as $\phi=\ell \Delta \beta$, where $\ell$ is the coiled fiber length and $\Delta \beta$ is the difference of the propagation constants of the two modes polarized along the two principal axes of the birefringent fiber. It can also be expressed as $\Delta \beta=k_{0} \Delta n=\frac{2 \pi}{\lambda} \cdot \Delta n$. Under small amount of variation in the wavelength $\Delta \lambda$, we have small amount of variation in the phase difference $\Delta \phi=\ell \Delta \lambda \frac{\mathrm{d}(\Delta \beta)}{\mathrm{d} \lambda}=\ell \Delta \lambda\left(-\frac{2 \pi}{\lambda^{2}} \Delta n\right)$. Therefore the refractive-index difference can be expressed as $\Delta n=-\frac{\Delta \phi}{\Delta \lambda} \frac{\lambda^{2}}{2 \pi \ell}$. As a result, we can obtain the mean value of the refractive-index difference of the coiled fiber through $\Delta n=-\frac{\Delta \phi}{\Delta \lambda} \frac{\lambda_{c}^{2}}{2 \pi \ell}$, where $\lambda_{c}$ is the central wavelength in operation [6,7]. The coiled single-mode optical fiber specifications and the $\Delta n$ measurement results under various bending radius values are listed in Table 1.

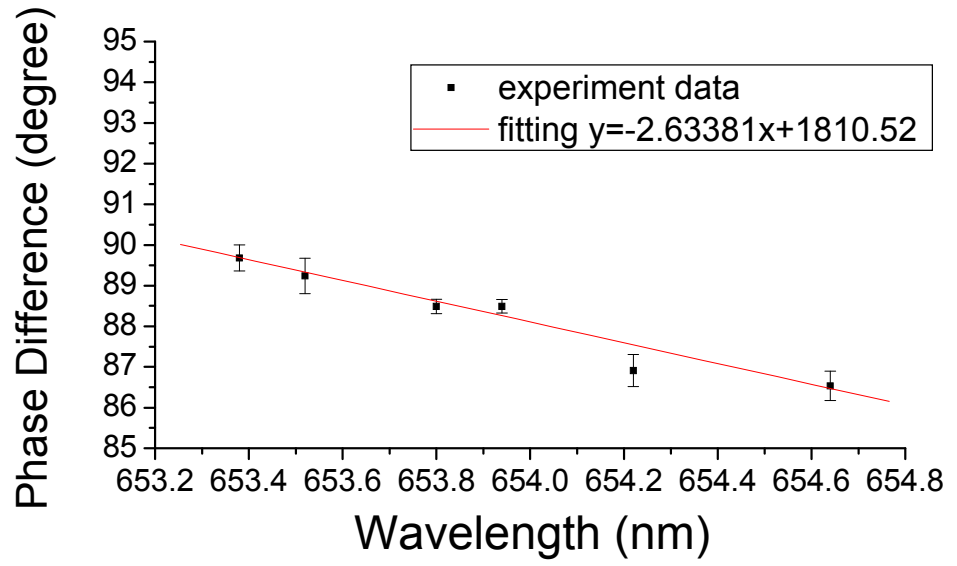

Figure 6. The measurement result of the phase difference of the two orthogonally polarized modes in a coiled single-mode optical fiber with a bending radius of $12.50 \mathrm{~mm}$ under various wavelengths. 
Table 1. The specifications and measurement results for a coiled single-mode fiber with various bending radius values.

\begin{tabular}{|c|c|c|c|c|c|}
\hline $\begin{array}{c}\text { Bending radius } \\
R \text { (mm) }\end{array}$ & $\begin{array}{c}\text { Coil number } \\
N\end{array}$ & $\begin{array}{c}\text { Bent fiber length } \\
\ell=2 \pi R N \quad(\mathrm{~mm})\end{array}$ & $\begin{array}{c}\text { Central wavelength } \\
\lambda_{c}(\mathrm{~nm})\end{array}$ & $\Delta \phi / \Delta \lambda \quad$ (degree /nm) & $\begin{array}{c}\text { Refractive-index } \\
\text { difference } \Delta n\end{array}$ \\
\hline 54.70 & 1 & 343.69 & 654.36 & -0.33860 & $1.172 \times 10^{-6}$ \\
\hline 29.95 & 2 & 376.36 & 654.22 & -1.05716 & $3.339 \times 10^{-6}$ \\
\hline 22.60 & 3 & 471.71 & 653.94 & -1.62878 & $4.542 \times 10^{-6}$ \\
\hline 15.18 & 3 & 286.04 & 653.94 & -2.26200 & $9.394 \times 10^{-6}$ \\
\hline 12.50 & 3 & 235.62 & 654.01 & -2.63381 & $1.328 \times 10^{-5}$ \\
\hline 9.85 & 3 & 185.67 & 654.60 & -3.48446 & $2.234 \times 10^{-5}$ \\
\hline 6.00 & 4 & 150.80 & 653.94 & -7.69265 & $6.060 \times 10^{-5}$ \\
\hline
\end{tabular}

Afterward we plot the measurement results of the refractive-index difference $\Delta n$ between the two orthogonally polarized modes in a coiled single-mode optical fiber as a function of the bending radius $R$ of the fiber coil, as shown in Figure 7. When we consider the fiber coil radius ranging from 6.00 to $22.60 \mathrm{~mm}$, the power of the fitting curve is -1.963 (error percentage $-1.85 \%$ ), well approaching the power -2 of the predicted curve in a square inverse law [9]. Nevertheless, when we consider the fiber coil radius ranging from 6.00 to $54.70 \mathrm{~mm}$, the power of the fitting curve is -1.7636 (error percentage $-11.82 \%$ ), slightly departing from the predicted power -2 . This reveals that the established optical system can determine precisely the refractive-index difference $\Delta n$ as small as $4.542 \times 10^{-6}$. If reduced to $\Delta n=$ $1.172 \times 10^{-6}$, the measurement result will begin to exhibit larger error, which is related to the intensity-variation detection limit of the CCD camera. Therefore the sensitivity of the refractive-index difference $\Delta n$ for this optical system is approximately at the order of $10^{-6}$.

(a)

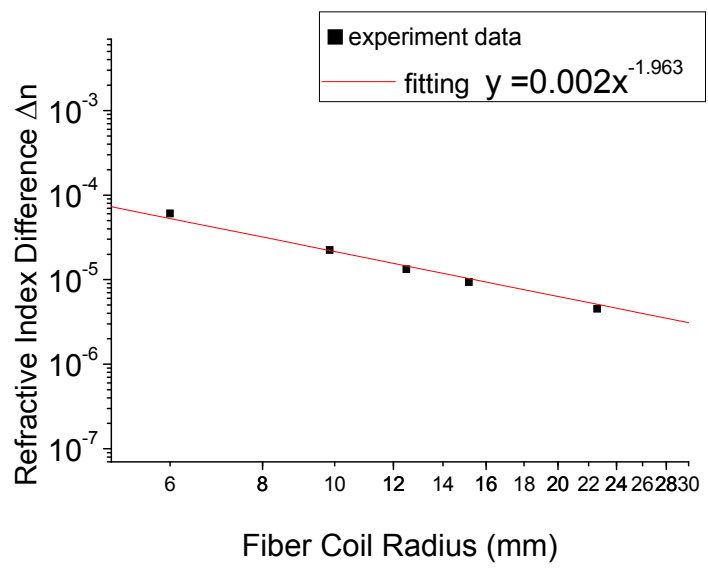

(b)

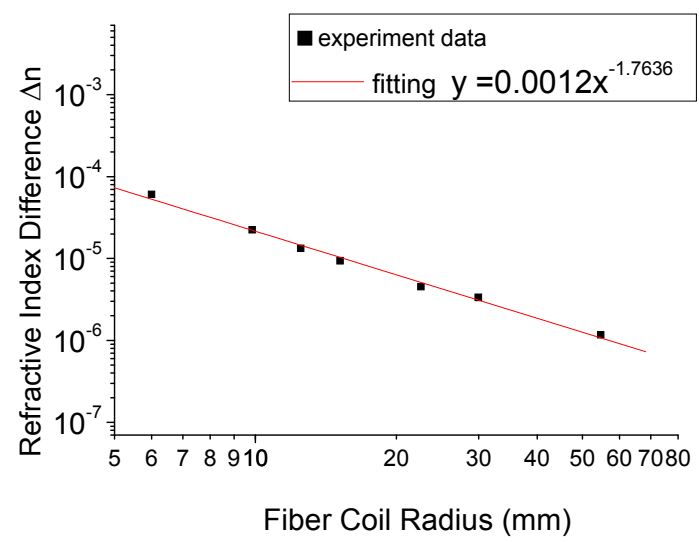

Figure 7. The measurement results of the refractive-index difference of a coiled single-mode optical fiber as a function of the bending radius of the fiber coil. The considered fiber coil radius ranges (a) from 6.00 to $22.60 \mathrm{~mm}$, and (b) from 6.00 to $54.70 \mathrm{~mm}$, respectively.

\section{CONCLUSIONS}

By wrapping a single-mode optical fiber around various sizes of cylindrical acrylic tubes, we can make a series of manually controllable birefringent optical fibers. The linearly polarized external-cavity feedback wavelength-tunable visible diode laser light is launched into the stressed birefringent fiber. By incorporating a Wollaston prism polarizer into the imaging system, we can simultaneously capture the fiber cross-sectional images of the two orthogonally polarized modes in the bent fiber core. Thus we can precisely obtain the ratio of the light intensities of these two modes and calculate their phase difference. By adjusting the diffraction grating's angle in the external-cavity feedback diode laser system, we can set the diode laser at various output wavelengths. In this way, we can measure the wavelength 
dependence of the phase difference between the two orthogonally polarized modes and then determine the refractive-index difference of the stressed birefringent fiber. Furthermore, by successively changing the size of the acrylic tube and repeating the above measurement, we can prove that the refractive-index difference of a coiled fiber varies with its bending radius in a square inverse law. The birefringence of a coiled single-mode optical fiber can be determined precisely by a home-made wavelength-tunable diode laser and a fiber mode-image analysis method, which compose an advanced teaching kit in the optics laboratory.

\section{ACKNOWLEDGEMENTS}

We acknowledge the financial support from the National Science Council, and Ministry of Science and Technology, Taiwan, through Projects NSC 100-2112-M-415-002-MY3, and MOST 103-2112-M-415-005, respectively.

\section{REFERENCES}

[1] Ulrich, R., Rashleigh, S. C. and Eickhoff, W., "Bending-induced birefringence in single-mode fibers," Optics Letters 5(6), 273-275 (1980).

[2] Aben, H., "Characteristic directions in optics of twisted birefringent media," Journal of the Optical Society of America A 3(9), 1414-1421 (1986).

[3] Kikuchi, K. and Okoshi, T., "Wavelength-sweeping technique for measuring the beat length of linearly birefringent optical fibers," Optics Letters 8(2), 122-123 (1983).

[4] Rashleigh, S. C., "Measurement of Fiber Birefringence by Wavelength Scanning: Effect of Dispersion," Optics Letters 8(6), 336-338 (1983).

[5] Sheu, Fang-Wen and Yang, Shu-Chun, "Using a wavelength tunable diode laser to measure the beat length of a birefringent fiber," in Education and Training in Optics and Photonics (ETOP), OSA Technical Digest Series (Optical Society of America, 2007), paper EMB3.

[6] Sheu, Fang-Wen and Chang, Ren-Sin, "Using External-cavity Feedback Wavelength-tunable Diode Laser and Image Analysis Technique to Measure the Characteristics of Birefringent Optical Fibers," The 17th OptoElectronics and Communications Conference (OECC 2012), Busan, Korea (2012 July), paper P1-28.

[7] Sheu, Fang-Wen and Cheng, Chang-Wen, "Using wavelength-tunable, reduced-coherence diode laser light to characterize birefringent optical fibers," The 12th Asia Pacific Physics Conference (APPC12), Chiba, Japan (2013 July), paper C3-1-O1.

[8] Sheu, Fang-Wen and Luo, Pei-Ling, "Development of a variable spectral-width, wavelength-tunable light source using a superluminescent diode with optical feedback," American Journal of Physics 76(8), 769-776 (2008).

[9] Chen, Chin-Lin, "Elements of Optoelectronics and Fiber Optics," Richard D. Irwin, USA (1996). 